Author affiliations appear at the end of this article.

Published online ahead of print at www.jco.org on February 8, 2016

Clinical Practice Guideline Committee approval: September 21, 2015.

Editor's note: This American Society of Clinical Oncology clinical practice guideline provides recommendations

based on the comprehensive review and analyses of the relevant literature for each recommendation. Additional information, which may include a data supplement with additional evidence tables, a methodology supplement, slide sets, clinical tools and resources, and links to patient information at www.cancer.net, is available at: $w w w . a s c o . o r g / g u i d e l i n e s$ and www.asco.org/guidelineswiki.

Authors' disclosures of potential conflicts of interest are found in the article online at www.jco.org. Author contributions are found at the end of this article.

Reprint requests: American Society of Clinical Oncology, 2318 Mill Road, Suite 800, Alexandria, VA 22314; guidelines@ asco.org

Corresponding author: American Society of Clinical Oncology, 2318 Mill Rd, Suite 800, Alexandria, VA 22314; e-mail: guidelines@asco.org.

C 2016 by American Society of Clinical Oncology

0732-183X/16/3410w-1134w/\$20.00

DOI: 10.1200/JCO.2015.65.2289

\title{
Use of Biomarkers to Guide Decisions on Adjuvant Systemic Therapy for Women With Early-Stage Invasive Breast Cancer: American Society of Clinical Oncology Clinical Practice Guideline
}

Lyndsay N. Harris, Nofisat Ismaila, Lisa M. McShane, Fabrice Andre, Deborah E. Collyar, Ana M. Gonzalez-Angulo, Elizabeth H. Hammond, Nicole M. Kuderer, Minetta C. Liu, Robert G. Mennel, Catherine Van Poznak, Robert C. Bast, and Daniel F. Hayes

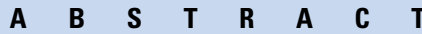

\section{Purpose}

To provide recommendations on appropriate use of breast tumor biomarker assay results to guide decisions on adjuvant systemic therapy for women with early-stage invasive breast cancer.

\section{Methods}

A literature search and prospectively defined study selection sought systematic reviews, metaanalyses, randomized controlled trials, prospective-retrospective studies, and prospective comparative observational studies published from 2006 through 2014. Outcomes of interest included overall survival and disease-free or recurrence-free survival. Expert panel members used informal consensus to develop evidence-based guideline recommendations.

\section{Results}

The literature search identified 50 relevant studies. One randomized clinical trial and 18 prospectiveretrospective studies were found to have evaluated the clinical utility, as defined by the guideline, of specific biomarkers for guiding decisions on the need for adjuvant systemic therapy. No studies that met guideline criteria for clinical utility were found to guide choice of specific treatments or regimens.

\section{Recommendations}

In addition to estrogen and progesterone receptors and human epidermal growth factor receptor 2, the panel found sufficient evidence of clinical utility for the biomarker assays Oncotype DX, EndoPredict, PAM50, Breast Cancer Index, and urokinase plasminogen activator and plasminogen activator inhibitor type 1 in specific subgroups of breast cancer. No biomarker except for estrogen receptor, progesterone receptor, and human epidermal growth factor receptor 2 was found to guide choices of specific treatment regimens. Treatment decisions should also consider disease stage, comorbidities, and patient preferences.

\section{J Clin Oncol 34:1134-1150. (c) 2016 by American Society of Clinical Oncology}

\section{INTRODUCTION}

This guideline provides evidence-based recommendations to practicing oncologists and other stakeholders on the appropriate use of breast tumor biomarker assay results to guide decisions on adjuvant systemic therapy for women with early-stage invasive breast cancer with known hormone receptor (estrogen and progesterone receptors [ER/PgRs]) and human epidermal growth factor receptor 2 [HER2]) status. In an era of great interest in personalized, precision medicine, the role of tumor biomarker assays in guiding clinical care has taken on even greater importance than in the past.

A prior update of the ASCO guideline on the use of tumor markers in breast cancer ${ }^{1}$ considered all indications or uses for biomarker assay results, which include screening, diagnosis, prognosis, and monitoring for recurrence or progression. Subsequently, ASCO collaborated with the College of American Pathologists (CAP) to publish and update guidelines on testing for HER $2^{2,3}$ and ER/PgR. ${ }^{4}$ Thus, to facilitate future updates in a rapidly developing field, the ASCO Breast Cancer 


\section{Use of Biomarkers to Guide Decisions on Adjuvant Systemic Therapy for Women With Early-Stage Invasive Breast Cancer: American Society of Clinical Oncology Clinical Practice Guideline}

\section{Guideline Questions}

- For women with early-stage invasive breast cancer and with known estrogen and progesterone receptor (ER/PgR) and human epidermal growth factor receptor 2 (HER2) status, which other biomarkers have demonstrated clinical utility to guide decisions on the need for adjuvant systemic therapy?

- For women with early-stage invasive breast cancer and with known ER/PgR and HER2 status, which additional biomarkers have demonstrated clinical utility to guide choice of specific drugs or regimens for adjuvant systemic therapy?

\section{Target Population}

Women with early-stage invasive breast cancer under consideration for adjuvant systemic therapy

\section{Target Audience}

Medical, surgical, and radiation oncologists; oncology nurses and physician assistants; pathologists; general practitioners; and patients

\section{Methods}

A Biomarker Expert Panel developed the clinical practice guideline recommendations on the basis of a systematic review of the medical literature.

\section{Key Points and Recommendations}

For women with early-stage invasive breast cancer and with known ER/PgR and HER2 status, which other biomarkers have demonstrated clinical utility to guide decisions on the need for adjuvant systemic therapy?

\section{Oncotype $D X$}

- If a patient has ER/PgR-positive, HER2-negative (node-negative) breast cancer, the clinician may use the 21-gene recurrence score (RS; Oncotype DX; Genomic Health, Redwood City, CA) to guide decisions on adjuvant systemic chemotherapy. Type: evidence based. Evidence quality: high. Strength of recommendation: strong.

- If a patient has ER/PgR-positive, HER2-negative (node-positive) breast cancer, the clinician should not use the 21-gene RS to guide decisions on adjuvant systemic chemotherapy. Type: evidence based. Evidence quality: intermediate. Strength of recommendation: moderate.

- If a patient has HER2-positive breast cancer or TN breast cancer, the clinician should not use the 21-gene RS to guide decisions on adjuvant systemic therapy. Type: informal consensus. Evidence quality: insufficient. Strength of recommendation: strong.

\section{EndoPredict}

- If a patient has ER/PgR-positive, HER2-negative (node-negative) breast cancer, the clinician may use the 12-gene risk score (EndoPredict; Sividon Diagnostics, Köln, Germany) to guide decisions on adjuvant systemic chemotherapy. Type: evidence based. Evidence quality: intermediate. Strength of recommendation: moderate.

- If a patient has ER/PgR-positive, HER2-negative (node-positive) breast cancer, the clinician should not use the 12-gene risk score (EndoPredict) to guide decisions on adjuvant systemic chemotherapy. Type: evidence based. Evidence quality: insufficient. Strength of recommendation: moderate.

- If a patient has HER2-positive breast cancer or TN breast cancer, the clinician should not use the 12-gene risk score (EndoPredict) to guide decisions on adjuvant systemic therapy. Type: informal consensus. Evidence quality: insufficient. Strength of recommendation: strong.

\section{MammaPrint}

- If a patient has ER/PgR-positive, HER2-negative (node-positive or node-negative) breast cancer, the clinician should not use the 70-gene assay (MammaPrint; Agendia, Irvine, CA) to guide decisions on adjuvant systemic chemotherapy. Type: evidence based. Evidence quality: intermediate. Strength of recommendation: moderate.

(continued on following page) 


\section{THE BOTTOM LINE (CONTINUED)}

- If a patient has HER2-positive breast cancer, the clinician should not use the 70-gene assay (MammaPrint) to guide decisions on adjuvant systemic therapy. Type: informal consensus. Evidence quality: low. Strength of recommendation: moderate.

- If a patient has TN breast cancer, the clinician should not use the 70-gene assay (MammaPrint) to guide decisions on adjuvant systemic therapy. Type: informal consensus. Evidence quality: insufficient. Strength of recommendation: strong.

\section{PAM50 risk of recurrence score}

- If a patient has ER/PgR-positive, HER2-negative (node-negative) breast cancer, the clinician may use the PAM50 risk of recurrence (ROR) score (Prosigna Breast Cancer Prognostic Gene Signature Assay; NanoString Technologies, Seattle, WA), in conjunction with other clinicopathologic variables, to guide decisions on adjuvant systemic therapy. Type: evidence based. Evidence quality: high. Strength of recommendation: strong.

- If a patient has ER/PgR-positive, HER2-negative (node-positive) breast cancer, the clinician should not use the PAM50-ROR to guide decisions on adjuvant systemic therapy. Type: evidence based. Evidence quality: intermediate. Strength of recommendation: moderate.

- If a patient has HER2-positive breast cancer, the clinician should not use the PAM50-ROR to guide decisions on adjuvant systemic therapy. Type: informal consensus. Evidence quality: insufficient. Strength of recommendation: strong.

- If a patient has TN breast cancer, the clinician should not use the PAM50-ROR to guide decisions on adjuvant systemic therapy. Type: informal consensus. Evidence quality: insufficient. Strength of recommendation: strong.

\section{Breast Cancer Index}

- If a patient has ER/PgR-positive, HER2-negative (node-negative) breast cancer, the clinician may use the Breast Cancer Index to guide decisions on adjuvant systemic therapy. Type: evidence based. Evidence quality: intermediate. Strength of recommendation: moderate.

- If a patient has ER/PgR-positive, HER2-negative (node-positive) breast cancer, the clinician should not use the Breast Cancer Index to guide decisions on adjuvant systemic therapy. Type: informal consensus. Evidence quality: insufficient. Strength of recommendation: strong.

- If a patient has HER2-positive breast cancer or TN breast cancer, the clinician should not use the Breast Cancer Index to guide decisions on adjuvant systemic therapy. Type: informal consensus. Evidence quality: insufficient. Strength of recommendation: strong.

\section{Mammostrat}

- If a patient has ER/PgR-positive, HER2-negative (node-positive or node-negative) breast cancer, the clinician should not use the five-protein assay (Mammostrat; Clarient, a GE Healthcare company, Aliso Viejo, CA) to guide decisions on adjuvant systemic therapy. Type: evidence based. Evidence quality: intermediate. Strength of recommendation: moderate.

- If a patient has HER2-positive breast cancer or TN breast cancer, the clinician should not use the five-protein assay (Mammostrat) to guide decisions on adjuvant systemic therapy. Type: informal consensus. Evidence quality: insufficient. Strength of recommendation: strong.

\section{Immunohistochemistry 4}

- If a patient has ER/PgR-positive, HER2-negative (node-positive or node-negative) breast cancer, the clinician should not use immunohistochemistry 4 (IHC4) to guide decisions on adjuvant systemic chemotherapy. Type: evidence based. Evidence quality: intermediate. Strength of recommendation: moderate.

- If a patient has HER2-positive breast cancer or TN breast cancer, the clinician should not use IHC4 to guide decisions on adjuvant systemic therapy. Type: informal consensus. Evidence quality: insufficient. Strength of recommendation: strong.

\section{Urokinase plasminogen activator and plasminogen activator inhibitor type 1}

- If a patient has ER/PgR-positive, HER2-negative (node-negative) breast cancer, the clinician may use urokinase plasminogen activator and plasminogen activator inhibitor type 1 to guide decisions on adjuvant systemic therapy. Type: evidence based. Evidence quality: high. Strength of recommendation: weak.

- If a patient has HER2-positive breast cancer or TN breast cancer, the clinician should not use urokinase plasminogen activator and plasminogen activator inhibitor type 1 to guide decisions on adjuvant systemic therapy. Type: informal consensus. Evidence quality: insufficient. Strength of recommendation: weak.

(continued on following page) 


\section{Circulating tumor cells}

- The clinician should not use circulating tumor cells to guide decisions on adjuvant systemic therapy. Type: evidence based. Evidence quality: intermediate. Strength of recommendation: strong.

\section{Tumor-infiltrating lymphocytes}

- If a patient has ER/PgR-positive, HER2-negative (node-positive or node-negative) breast cancer, the clinician should not use tumorinfiltrating lymphocytes to guide decisions on adjuvant systemic therapy. Type: informal consensus. Evidence quality: insufficient. Strength of recommendation: strong.

- If a patient has HER2-positive breast cancer or TN breast cancer, the clinician should not use tumor-infiltrating lymphocytes to guide decisions on adjuvant systemic therapy. Type: evidence based. Evidence quality: intermediate. Strength of recommendation: strong.

\section{Protein encoded by the MKI67 gene}

- Protein encoded by the MKI67 gene labeling index by IHC should not be used to guide choice on adjuvant chemotherapy. Type: evidence based. Evidence quality: intermediate. Strength of recommendation: moderate.

\section{Extended endocrine therapy}

- If a patient has ER/PgR-positive, HER2-negative (node-negative) breast cancer and has had 5 years of endocrine therapy without evidence of recurrence, the clinician should not use multiparameter gene expression or protein assays (Oncotype DX, EndoPredict, PAM50, Breast Cancer Index, or IHC4) to guide decisions on extended endocrine therapy. Type: evidence based. Evidence quality: intermediate. Strength of recommendation: moderate.

For women with early-stage invasive breast cancer and with known ER/PgR and HER2 status, which additional biomarkers have demonstrated clinical utility to guide choice of specific drugs or regimens for adjuvant systemic therapy?

\section{Tamoxifen}

- The clinician should not use CYP2D6 polymorphisms to guide adjuvant endocrine therapy selection. Type: evidence based. Evidence quality: intermediate. Strength of recommendation: moderate.

- The clinician should not use p27 expression by IHC to guide adjuvant endocrine therapy selection. Type: informal consensus. Evidence quality: low. Strength of recommendation: strong.

\section{Aromatase inhibitors}

- The clinician should not use protein encoded by the MKI67 gene labeling index by IHC to guide adjuvant endocrine therapy selection. Type: evidence based. Evidence quality: intermediate. Strength of recommendation: moderate.

\section{Taxanes}

- The clinician should not use microtubule-associated protein Tau mRNA expression or mRNA expression by IHC to guide adjuvant chemotherapy selection. Type: evidence based. Evidence quality: intermediate. Strength of recommendation: moderate.

- The clinician should not use HER1/epidermal growth factor receptor expression by IHC to guide adjuvant chemotherapy selection. Type: evidence based. Evidence quality: low. Strength of recommendation: moderate.

\section{Anthracyclines}

- The clinician should not use TOP2A gene amplification or TOP2A protein expression by IHC to guide adjuvant chemotherapy selection. Type: evidence based. Evidence quality: high. Strength of recommendation: moderate.

- The clinician should not use HER2 and TOP2A gene coamplification; CEP17 duplication; or TIMP-1, FOXP3, or p53 to guide adjuvant chemotherapy selection. Type: evidence based. Evidence quality: intermediate. Strength of recommendation: moderate.

(continued on following page) 


\section{Trastuzumab}

- If a patient has HER2-positive breast cancer, the clinician should not use PTEN to guide adjuvant therapy selection. Type: evidence based. Evidence quality: intermediate. Strength of recommendation: moderate.

- If a patient has HER2-positive breast cancer, the clinician should not use soluble HER2 levels to guide selection of type of adjuvant therapy. Type: evidence based. Evidence quality: low. Strength of recommendation: moderate.

\section{Additional Resources}

More information, which includes Data Supplements with additional specific recommendations (Data Supplement Tables S2 and S3), evidentiary requirement table (Data Supplement Tables S4 and S5), a Methodology Supplement with information about evidence quality and strength of recommendations, slide sets, and clinical tools and resources, is available at www.asco.org/guidelines/ adjuvantbreastmarkers and www.asco.org/guidelineswiki. Patient information is available at www.cancer.net.

Guidelines Advisory Group and Clinical Practice Guidelines Committee determined that guidelines on additional breast cancer biomarkers should focus on single uses or indications (see Use Context) and select their use to guide decisions on systemic therapy, both designated as the highest priority topics. This prioritization resulted in this guideline for women with early-stage invasive breast cancer and another guideline on systemic therapy for women with metastatic breast cancer. ${ }^{4 a}$

\section{Objective Process to Develop and Evaluate Guidelines for Tumor Biomarker Tests}

Many reviews and commentaries have discussed the nature of evidence required to support conclusions that the use of biomarker test results to guide treatment decisions improves health outcomes for patients with a malignancy. ${ }^{5-12}$ The present guideline and the companion guideline on systemic therapy for women with metastatic breast cancer have endorsed the semantic descriptions first proposed by the Evaluation of Genomic Applications in Practice and Prevention Working Group ${ }^{13,14}$ and subsequently adapted by the Institute of Medicine Committee on Evolution of Translational Omics. ${ }^{15}$ As described in this guideline and in Data Supplement 1, Table S1, the working group defined three criteria for evidence: analytic validity, clinical validity, and clinical utility. These new criteria are more rigorous than those applied in the 2007 ASCO biomarker guideline. ${ }^{1}$

The Biomarker Expert Panel recognized that the term biomarker refers to the general biologic or molecular condition that distinguishes one patient group from another. However, various tests may measure one biomarker, and they may have different analytic and clinical validities; thus, the clinical utility for each test also may differ. For example, instead of recommending that HER2 be evaluated in all patients with breast cancer, the ASCO/CAP HER2 testing guidelines specifically recommended that individual assays of amplification or overexpression be used. ${ }^{3,16}$ Therefore, to the extent possible, the panel evaluated each tumor biomarker assay for a given biomarker and made recommendations for each specific test. In the same manner as the ASCO/CAP HER2 Testing Update Committee, the Biomarker Expert Panel evaluated, in some cases, a class of biomarker tests for a single analyte; in other cases, the panel evaluated specific commercially available tests, especially those that were composed of multianalyte signatures. These decisions were guided by the available literature on the clinical utility of the biomarker in question. The following sections describe more carefully the terms use context, analytic and clinical validity, and clinical utility as they were applied to each biomarker by the expert panel.

\section{Use Context}

A tumor biomarker test might be used in one of many contexts. These use contexts include risk categorization (eg, determination of germline mutations as a marker of susceptibility), screening, differential diagnosis, prognosis, prediction, or monitoring. For this guideline, the panel considered only prognosis and prediction in patients with newly diagnosed, nonmetastatic, primary breast cancer. Prognosis was defined as an indication of future risk of an event (recurrence, distant metastases, or death) independent of the effect of prior or anticipated therapy. Prediction was defined as the ability of a specific biomarker to indicate the likelihood of benefit from a particular therapy or a class of agent (eg, endocrine, biologic, or chemotherapy).

\section{Analytic Validity}

Analytic validity refers to the accuracy, reliability, and reproducibility of the assay as demonstrated by preanalytic, technical, and scoring or interpretation methods. The panel reviewed the analytic validity of a specific assay for a biomarker when there was evidence for clinical utility, and a recommendation for clinical use was considered. This information is reviewed in Data Supplement 1. Of note, these data often are not available and most often are provided when the tumor biomarker test has been manufactured for commercial purposes.

\section{Clinical Validity}

Clinical validity refers to the ability of a tumor biomarker test to divide one population into two or more groups that differ either biologically or clinically. For example, a tumor biomarker test has 
clinical validity if a group of patients with early-stage breast cancer is found to have a worse disease-free survival (DFS) or overall survival (OS) if their tumor is positive for the marker compared with those that are negative. Clinical validity often is illustrated in Kaplan-Meier curves and expressed as relative or proportional differences in outcomes in accordance with marker status, with associated measures of uncertainty or likelihood that the differences are due to chance alone (eg, confidence limits, $P$ values). Although it is unlikely that a tumor biomarker test would have clinical utility, if the biomarker does not have clinical validity, clinical validity alone is insufficient to recommend that the test be used to guide treatment decisions.

\section{Clinical Utility}

A biomarker-based test is judged to have clinical utility if use of the test is associated with a favorable balance of benefits to harms compared with treatment of the patient in the absence of the biomarker test result. ${ }^{8,9,13}$ Benefits may include improvement in survival end points such as event-free survival, DFS, progressionfree survival, or OS. A new biomarker test must be shown to contribute clinically useful information beyond that already provided by clinical or pathologic indicators in standard use, unless the new test can provide equivalent information at lower cost, less invasively, or with less inconvenience or risk. The magnitude of the benefit must be clinically meaningful and outweigh risks, costs, and/or inconvenience associated with use of the test.

The degree of benefit required to recommend for or against a treatment must be tempered with clinical judgment and patient perspective. For example, when one considers whether to recommend adjuvant chemotherapy for a patient with early-stage breast cancer, the odds of distant recurrence over the succeeding 10 years for a patient with a node-positive cancer that is negative for ER, PgR, and HER2 exceeds 50\% in the absence of adjuvant systemic therapy. An overview by the Early Breast Cancer Trialists Collaborative Group suggested that the reduction of risk of recurrence (ROR) with adjuvant chemotherapy is at least $30 \%{ }^{17}$ Therefore, adjuvant chemotherapy would improve a patient's odds of distant, incurable recurrence by approximately $15 \%$ to $20 \%$ (one-third of the $50 \%$ to $60 \%$ initial risk). Several studies have suggested that the odds of fatal, life-threatening, or permanent life-changing toxicities are at least $2 \%$ to $3 \%$ in healthy women who participate in prospective trials. Therefore, one might reasonably assume that the number of women who will benefit from the adjuvant chemotherapy will far exceed the number who will be harmed; therefore, one would probably recommend its use in this situation.

Consider a patient with a grade 1, ER/PgR-positive, HER2negative (node-negative) breast cancer. Her odds of recurrence over 10 years are approximately $10 \%$ to $15 \%$. Let us assume that she would receive adjuvant endocrine therapy, which will reduce these odds by approximately one-third to one-half and leave approximately $10 \%$ at residual risk. By applying the same assumption that adjuvant chemotherapy will further reduce her ROR by approximately one-third, a recommendation for treatment to similar patients will only benefit $2 \%$ to $3 \%$, which is roughly the same number who will be harmed.

One must then consider other issues, such as the higher risk of serious toxicities associated with comorbidities and the relatively lesser benefits in terms of long-term breast cancer-related survival in elderly women. The clinician and patient must decide together in each case whether treatment is worthwhile. Accurate tumor biomarker assays can aid in this discussion.

Reliable evidence. As with new therapeutic agents, demonstration of clinical utility for a biomarker test must be accomplished by using high levels of evidence obtained from well-designed and -executed studies. The panel followed the proposals made by Simon et $\mathrm{al}^{9}$ for determining whether a biomarker test has clinical utility. Ideally, clinical utility of a tumor biomarker test would be generated in a prospective trial in which evaluation of the clinical utility of the tumor biomarker test is the primary objective. Several authors have proposed recommendations for such trial designs. ${ }^{18,19}$ In a hierarchal ranking of types of studies to determine clinical utility of tumor biomarker tests, Simon et $\mathrm{al}^{9}$ suggested that such truly prospective trials should be designated as category A.

Alternatively, clinical utility of a tumor biomarker test might be determined by using archived specimens in what Simon et $\mathrm{al}^{9}$ termed prospective-retrospective studies. However, these authors carefully stipulated that studies of convenience in which the collection of the specimens was not prospectively dictated and treatment and outcomes determination were not prospectively proscribed or defined are associated with substantial bias and unlikely to be sufficient to determine clinical utility. In contrast, they suggested that prospectiveretrospective studies are best performed by using archived specimens collected and stored within the context of a prospective trial to address the use context in which the biomarker test might be applied. Simon et al recommended that such prospective-retrospective studies be designated as category $\mathrm{B}$ (Table 1 ).

Simon et $\mathrm{al}^{9}$ further suggested that clinical utility be considered level of evidence 1 if one category A study was available that showed meaningful differences in outcome on the basis of the biomarker. They also suggested that at least two category B studies were required, both by showing similar results, to achieve level of evidence 1 status.

Additional considerations. When the panel considered each of the tumor biomarker assay tests, the use context, analytic validity, clinical validity, and clinical utility were considered. For the use context of estimating prognosis to consider whether adjuvant chemotherapy should be administered, the panel recommended

Table 1. Requirements for a Marker-Based Test to Reach Level IB Evidence of Clinical Utility on the Basis of Prospective-Retrospective Studies

\section{Requirements}

1. Adequate amounts of archived specimen must be available from enough patients from a prospective trial (which for predictive factors should generally be a randomized design) for analyses to have adequate statistical power and for the patients included in the evaluation to be clearly representative of the patients in the trial.

2. The marker-based test should be analytically and preanalytically validated for use with archived specimens.

3. The plan for marker evaluation should be completely specified in writing before the performance of marker assays on archived specimens and should be focused on the evaluation of a completely defined markerbased test.

4. The results from archived specimens should be validated by using specimens from one or more similar, but separate studies.

NOTE. Adapted from Simon et al. ${ }^{9}$ 
use of a tumor biomarker assay if high levels of evidence suggest that it identifies a group of patients for whom the absolute benefit of adjuvant chemotherapy could not exceed $2 \%$ to $3 \%$, which is roughly equal to the risk of fatal, life-threatening, or permanently changing toxicities. For other use contexts, the panel's considerations are noted in the appropriate section.

The panel reached a consensus for each biomarker test by applying the specified levels of evidence criteria. However, some subjective judgment was required to reach consensus on whether the observed magnitude of biomarker effect was sufficiently large to be clinically meaningful and whether the statistical evidence was sufficient. In situations where multiple studies that addressed the same biomarker yielded inconsistent findings or when the individual studies in a collection were weak (eg, some individual studies did not reach conventional statistical significance criteria) but all pointed in a consistent direction, the panel decided whether clinical utility had been established on the basis of the totality of the evidence. Additional challenges included the lack of information reported in many published tumor marker studies. For example, many published tumor marker studies did not report whether the evaluation plan for the marker was completely specified in advance and often provided little or no detail about whether the biomarker assay was analytically validated. Panel members used their expertise (clinical, laboratory, and statistical) and clinical experience to aid in reaching consensus.

\section{GUIDELINE QUESTIONS}

This clinical practice guideline addresses two clinical questions. For women with early-stage invasive breast cancer and with known ER/ PgR and HER2 status, which additional biomarkers have demonstrated clinical utility to guide decisions with regard to the need for adjuvant systemic therapy, and which biomarkers guide the choice of specific drugs or regimens for adjuvant systemic therapy?

\section{METHODS}

\section{Guideline Development Process}

The recommendations were developed by a multidisciplinary expert panel that convened by teleconference, Webinar, or in person and corresponded through e-mail. Biomarker selection was based on direct application to the clinical questions. For example, BRCA1 and BRCA2 germline mutations were not considered for this guideline because they have no direct application to choice of adjuvant therapy at this time. All authors evaluated the evidence and contributed to the development of the guideline by critically reviewing and approving the guideline recommendations. The penultimate version of the guideline was submitted to Journal of Clinical Oncology for editorial review and consideration for publication. All ASCO guidelines are ultimately reviewed and approved by the expert panel and the ASCO Clinical Practice Guideline Committee before publication.

The expert panel developed its recommendations on the basis of evidence identified through online searches of Medline and the Cochrane Library (from January 2006 through August 2014 to overlap with the search end date for the 2007 guideline update on tumor markers in breast cancer ${ }^{1}$ ), which were complemented by members' additional suggestions of articles missing from the original searches. Data Supplement 2 contains full details on the search string. A combined search was conducted for this guideline and for a similar guideline on the use of biomarkers to guide decisions on systemic therapy in metastatic breast cancer, with articles selected for each guideline's systematic review in accordance with the patient population studied.

The articles that met the following criteria for population were selected for inclusion in the systematic review: women with early-stage invasive breast cancer under consideration for adjuvant systemic therapy, with separate subquestions and analyses on patient groups with ER/PgRpositive, HER2-negative disease; HER2-positive disease (ER positive or negative); and triple-negative (TN) disease (ER negative, PgR negative, and HER2 negative). Likewise, articles were selected that reported rigorously conducted systematic reviews (with or without meta-analyses), randomized clinical trials (RCTs), retrospective biomarker analyses of samples from completed prospective RCTs, or prospective observational studies that directly compared outcomes of treatment decisions made on the basis of assay results, with outcomes of treatment decisions made regardless of assay results.

Articles were excluded from the systematic review if they were meeting abstracts not subsequently published in peer-reviewed journals; editorials, commentaries, letters, news articles, case reports, or narrative reviews; and articles not published in English. Before publication, an updated search was completed with the date range of August 2014 to September 2015. No new evidence was found that would have altered the present recommendations.

The guideline recommendations were crafted, in part, by using the GLIDES (Guidelines Into Decision Support) methodology and accompanying BridgeWiz software (Yale University, New Haven, CT). In addition, a guideline review was conducted to determine ease of implementation. Revisions were made to clarify recommended actions for clinical practice. Ratings for the type and strength of recommendation, evidence, and potential bias are provided with each recommendation in the Data Supplements. Detailed information about the methods used to develop this guideline is available in the Methodology Supplement at www.asco.org/ guidelines/adjuvantbreastmarkers and www.asco.org/guidelineswiki, which includes an overview (eg, panel composition, development process, revision dates), literature search and data extraction, the recommendation development process, and quality assessment.

\section{Guideline Disclaimer}

The clinical practice guidelines and other guidance published herein are provided by ASCO to assist providers in clinical decision making. The information herein should not be relied on as complete or accurate, nor should it be considered as inclusive of all proper treatments or methods of care or as a statement of the standard of care. With the rapid development of scientific knowledge, new evidence may emerge between the time information is developed and the time it is published or read. The information is not continuously updated and may not reflect the most recent evidence. The information addresses only the topics specifically identified herein and is not applicable to other interventions, diseases, or stages of disease. This information does not mandate any particular course of medical care. Furthermore, the information is not intended to substitute for the independent professional judgment of the treating provider because it does not account for individual variation among patients. Recommendations reflect high, moderate, or low confidence in the net effect of a given course of action. The use of such words as must, must not, should, and should not indicates that a course of action is recommended or not recommended for either most or many patients, but latitude exists for the treating physician to select other courses of action in individual cases. In all cases, the selected course of action should be considered by the treating provider in the context of treating the individual patient. Use of the information is voluntary. ASCO provides this information on an as-is basis and makes no warranty, express or implied, with regard to the information. ASCO specifically disclaims any warranties of merchantability or fitness for a particular use or purpose. ASCO assumes no responsibility for any injury or damage to persons or property that arises out of or are related to any use of this information or for any errors or omissions. 


\section{Guideline and Conflicts of Interest}

The expert panel was assembled in accordance with the ASCO Conflict of Interest Policy Implementation for Clinical Practice Guidelines (summarized at www.asco.org/rwc). Members of the panel completed the ASCO disclosure form, which requires general disclosure of financial and other interests relevant to the subject matter of the guideline and includes relationships with commercial entities that are reasonably likely to experience a direct regulatory or commercial impact as a result of promulgation of the guideline. Categories for disclosure include employment relationships, consulting arrangements, stock and other ownership interests, speakers' bureaus, honoraria, research funding, intellectual property interests, and expert testimony. In accordance with the procedures, the majority of the members of the panel did not have any such conflicts of interest to disclose.

\section{RESULTS}

Fifty studies comprise the evidence base. ${ }^{20-69}$ They included three meta-analyses, ${ }^{47,57,59}$ one $\mathrm{RCT},{ }^{42} 38$ prospective-retrospective studies, ${ }^{20-27,34-36,38-41,43,45,46,48-56,58,60-69}$ three prospective comparative observational studies, ${ }^{33,37,44}$ and five retrospective observational studies. ${ }^{28-32}$ Twelve studies ${ }^{20,22,24-26,34-39,42}$ showed evidence of clinical utility and form the evidentiary basis for the guideline recommendations. The remaining 38 studies ${ }^{21,23,27-33,40,41,43-69}$ that did not focus on the evaluation of clinical utility were used to inform a recommendation against the use of other biomarkers identified.

The primary outcomes in most of these studies were DFS, recurrence-free survival, and OS. None of the included studies evaluated adverse outcomes of biomarker testing. In addition, no studies reported on changes in quality-of-life outcomes attributable to biomarker testing.

\section{RECOMMENDATIONS}

For each biomarker or assay listed herein, the literature was reviewed by using the parameters dictated in the guideline. When the biomarker in question was considered to have clinical utility, the analytic and clinical validity were reviewed in detail. Review of the literature for each biomarker or assay is provided in Data Supplement 3.

\section{CLINICAL QUESTION 1}

For women with early-stage invasive breast cancer and with known ER/PgR and HER2 status, which other biomarkers have demonstrated clinical utility to guide decisions on the need for adjuvant systemic therapy?

\section{Recommendation 1.1}

If a patient has ER/PgR-positive, HER2-negative (node-negative) breast cancer, the clinician may use the 21-gene recurrence score (RS; Oncotype DX; Genomic Health, Redwood City, CA) to guide decisions on adjuvant systemic chemotherapy. Type: evidence based. Evidence quality: high. Strength of recommendation: strong.

Clinical interpretation of literature review. The 21-gene RS assists with the decision of adjuvant chemotherapy in patients with ER/PgR-positive, HER2-negative (node-negative) breast cancer. $^{20-22}$ Chemotherapy is indicated in patients with a high RS and is not indicated in patients with a low RS. Recommendations for adjuvant chemotherapy in patients with an intermediate RS may be determined by TAILORx (Trial Assigning Individualized Options for Treatment; ClinicalTrials.gov identifier: NCT00310180).

\section{Recommendation $\mathbf{1 . 2}$}

If a patient has ER/PgR-positive, HER2-negative (nodepositive) breast cancer, the clinician should not use the 21-gene RS to guide decisions on adjuvant systemic chemotherapy. Type: evidence based. Evidence quality: intermediate. Strength of recommendation: moderate.

Clinical interpretation of literature review. The 21-gene RS might identify patients with ER/PgR-positive, HER2-negative (node-positive) breast cancer for whom chemotherapy might not be recommended on the basis of either prognosis (that the patient does not need chemotherapy) or prediction (that chemotherapy might have little or no benefit). ${ }^{23}$ Although the 21-gene RS is prognostic in patients with node-positive disease (patients with low RS have a better prognosis than patients with high RS), patients with node-positive disease but low RS have a worse prognosis than patients with node-negative, low RS disease. This different baseline prognosis for node-positive compared with node-negative disease may alter the balance of prognostic and predictive factors that lead to an overall recommendation to withhold or administer chemotherapy. For node-positive breast cancer, one must simultaneously consider the prognostic effects of the number of positive nodes and the 21-gene RS as well as any predictive value of the 21-gene RS.

The panel identified two main contexts in which a recommendation to withhold chemotherapy for patients with node-positive breast cancer might be considered. One context is that of a subgroup of patients with limited node-positive disease (one positive lymph node with a small deposit of cancer) for whom the prognostic role of the 21-gene RS might be similar to that for patients with node-negative disease. The panel also considered whether there might be a group of patients with more extensive nodal involvement, but for whom the predictive role of the 21-gene RS could identify whether chemotherapy would be ineffective, even though the patients' prognosis is unsatisfactory. However, the panel believed that more data are needed to determine the specific combinations of axillary nodal disease extent and RS that would define these patient subgroups.

The panel recognized that data from the Southwest Oncology Group (SWOG) S8814 and Arimidex, Tamoxifen, Alone or in Combination (ATAC) trials suggest that patients with only one to three positive nodes may have similar prognoses to those with negative nodes. ${ }^{22,23}$ However, the panel could not rule out the possibility of additional variation in prognosis due to number of positive nodes and did not reach consensus on withholding chemotherapy for patients with one or two positive axillary micrometastases and low RS on the basis of prognosis rather than prediction. The majority of panel members believed that additional high levels of evidence are necessary to recommend the application of this assay for that use context.

With regard to the predictive role of the 21-gene RS for chemotherapy, several studies suggested that cancers with high ER/ PgR and low HER2 and protein encoded by the MKI67 gene (Ki- 
67) might be less chemosensitive than those with low ER/PgR and high HER2 and Ki-67. ${ }^{70}$ Indeed, data generated from the National Surgical Adjuvant Breast and Bowel Project trial B20 suggested that adjuvant cyclophosphamide, methotrexate, and fluorouracil chemotherapy is ineffective in patients with node-negative disease and low RS, whereas it is effective in those with high RS. Likewise, the data from SWOG S8814 in patients with node-positive disease were similar. However, the B20 data are confounded by the data set originally used to generate the 21-gene RS algorithm. The results from SWOG S8814 must be considered hypothesis generating because the number of samples analyzed in each RS subgroup was small, there was no additional prediction beyond 5 years, and the risks of systemic recurrence continues to be high for patients with node-positive disease.

Because widespread use of adjuvant chemotherapy has had such a profound effect on reducing breast cancer mortality, ${ }^{21,23}$ the panel believed that clinicians must take a cautious approach to withholding it from patients with node-positive disease. However, there may be some patients for whom the relative benefit of chemotherapy does not justify the risk of toxicity. The RxPONDER trial ( $\mathrm{Rx}$ for Positive Node, Endocrine Responsive Breast Cancer; ClinicalTrials.gov identifier: NCT01272037) currently is accruing patients to answer this question and to identify the cutoff of the RS for which chemotherapy is beneficial for patients with one to three positive nodes. The Data Supplements provide more detailed discussion on this issue.

\section{Recommendation 1.3}

If a patient has HER2-positive breast cancer or TN breast cancer, the clinician should not use the 21-gene RS (Oncotype DX) to guide decisions on adjuvant systemic therapy. Type: informal consensus. Evidence quality: insufficient. Strength of recommendation: strong.

Clinical interpretation of literature review. Oncotype DX was developed for ER/PgR-positive breast cancer. The data do not support the use of Oncotype DX in HER2-positive breast cancer and $\mathrm{TN}$ breast cancer.

\section{Recommendation 1.4}

If a patient has ER/PgR-positive, HER2-negative (node-negative) breast cancer, the clinician may use the 12-gene risk score (EndoPredict; Sividon Diagnostics, Köln, Germany) to guide decisions on adjuvant systemic chemotherapy. Type: evidence based. Evidence quality: intermediate. Strength of recommendation: moderate.

Clinical interpretation of literature review. EndoPredict assists with the decision of whether to administer adjuvant chemotherapy in patients with ER/PgR-positive, HER2-negative (node-negative) breast cancer because it identifies patients with good outcomes on endocrine therapy alone at 10 years of follow-up. ${ }^{24-26}$

\section{Recommendation 1.5}

If a patient has ER/PgR-positive, HER2-negative (nodepositive) breast cancer, the clinician should not use the 12-gene risk score (EndoPredict) to guide decisions on adjuvant systemic chemotherapy. Type: evidence based. Evidence quality: insufficient. Strength of recommendation: moderate.
Clinical interpretation of literature review. Data do not support the use of EndoPredict in ER/PgR-positive, HER2-negative (nodepositive) breast cancer. ${ }^{27}$

\section{Recommendation 1.6}

If a patient has HER2-positive breast cancer or TN breast cancer, the clinician should not use the 12-gene risk score (EndoPredict) to guide decisions on adjuvant systemic therapy. Type: informal consensus. Evidence quality: insufficient. Strength of recommendation: strong.

Clinical interpretation of literature review. EndoPredict was developed for ER/PgR-positive breast cancer. No data support the use of EndoPredict in HER2-positive breast cancer or TN breast cancer.

\section{Recommendation 1.7}

If a patient has ER/PgR-positive, HER2-negative (nodepositive or node-negative) breast cancer, the clinician should not use the 70-gene assay (MammaPrint; Agendia, Irvine, CA) to guide decisions on adjuvant systemic chemotherapy. Type: evidence based. Evidence quality: intermediate. Strength of recommendation: moderate.

Clinical interpretation of literature review. MammaPrint has been shown to have prognostic value in ER/PgR-positive breast cancer. $^{28-30,32,33}$ However, the panel could not determine whether the assay identified a group of patients with either ER-positive or -negative, or node-positive or -negative, early-stage breast cancer for whom chemotherapy is either not required (prognosis) or not effective (prediction). Therefore, the panel does not recommend that the 70-gene assay be used to decide whether a patient should receive adjuvant chemotherapy. The panel awaits results of the prospectively conducted MINDACT (Microarray in NodeNegative and 1 to 3 Positive Lymph Node Disease May Avoid Chemotherapy; ClinicalTrials.gov identifier: NCT00433589) study, which will clarify these areas.

\section{Recommendation 1.8}

If a patient has HER2-positive breast cancer, the clinician should not use the 70-gene assay (MammaPrint) to guide decisions on adjuvant systemic therapy. Type: informal consensus. Evidence quality: low. Strength of recommendation: moderate.

Clinical interpretation of literature review. MammaPrint has prognostic value in HER2-positive breast cancer. ${ }^{31}$ However, the panel does not consider a 10 -year distant DFS of $84 \%{ }^{31}$ sufficiently favorable to omit chemotherapy from an adjuvant regimen and concluded that the data do not support use of the 70-gene assay to decide whether a patient with HER2-positive breast cancer should receive adjuvant chemotherapy.

\section{Recommendation $\mathbf{1 . 9}$}

If a patient has TN breast cancer, the clinician should not use the 70-gene assay (MammaPrint) to guide decisions on adjuvant systemic therapy. Type: informal consensus. Evidence quality: insufficient. Strength of recommendation: strong.

Clinical interpretation of literature review. MammaPrint has been shown to have prognostic value in ER/PgR-negative breast cancer; however, no data are available in patients with TN breast 
cancer. Given the substantial benefit of chemotherapy in this group of patients for whom no other therapies (endocrine therapy, anti-HER2) are available, the panel believed strongly that the 70-gene assay should not be used to guide clinical decisions in patients with TN breast cancer. Future recommendations about this test and adjuvant chemotherapy will be informed as part of the MINDACT study.

\section{Recommendation 1.10}

If a patient has ER/PgR-positive, HER2-negative (nodenegative) breast cancer, the clinician may use the PAM50 risk of recurrence (ROR) score (Prosigna Breast Cancer Prognostic Gene Signature Assay; NanoString Technologies, Seattle, WA) in conjunction with other clinicopathologic variables to guide decisions on adjuvant systemic therapy. Type: evidence based. Evidence quality: high. Strength of recommendation: strong.

Clinical interpretation of literature review. The PAM50-ROR test assists with the decision about adjuvant therapy in patients with ER/PgR-positive, HER2-negative (node-negative) breast cancer. ${ }^{34-36}$ Chemotherapy should be considered for patients in the PAM50 high-risk group, but is not indicated for patients in the low-risk group. Future studies are needed to inform recommendations about adjuvant chemotherapy in patients with an intermediate PAM50-ROR.

\section{Recommendation 1.11}

If a patient has ER/PgR-positive, HER2-negative (node-positive) breast cancer, the clinician should not use the PAM50-ROR (Prosigna Breast Cancer Prognostic Gene Signature Assay) to guide decisions on adjuvant systemic therapy. Type: evidence based. Evidence quality: intermediate. Strength of recommendation: moderate.

Clinical interpretation of literature review. Data are inconsistent with regard to choice of a cut point on the PAM50-ROR test to define ER/PgR-positive, HER2-negative (node-positive) breast cancer for which the ROR is sufficiently low to recommend against adjuvant systemic therapy. Therefore, more data are required to determine whether PAM50-ROR can be used with confidence in guiding the use of adjuvant systemic therapy for ER/PgR-positive, HER2-negative (node-positive) breast cancer.

\section{Recommendation 1.12}

If a patient has HER2-positive breast cancer, the clinician should not use the PAM50-ROR to guide decisions on adjuvant systemic therapy. Type: informal consensus. Evidence quality: insufficient. Strength of recommendation: strong.

Clinical interpretation of literature review. No data support the use of PAM50-ROR in HER2-positive breast cancer.

\section{Recommendation 1.13}

If a patient has TN breast cancer, the clinician should not use the PAM50-ROR to guide decisions on adjuvant systemic therapy. Type: informal consensus. Evidence quality: insufficient. Strength of recommendation: strong.

Clinical interpretation of literature review. The studied clinical trial populations excluded TN breast cancer; therefore, no data support the use of PAM50-ROR in TN breast cancer.

\section{Recommendation 1.14}

If a patient has ER/PgR-positive, HER2-negative (nodenegative) breast cancer, the clinician may use the Breast Cancer Index (bioTheranostics, San Diego, CA) to guide decisions on adjuvant systemic therapy. Type: evidence based. Evidence quality: intermediate. Strength of recommendation: moderate.

Clinical interpretation of literature review. The Breast Cancer Index assists with the decision for adjuvant chemotherapy in patients with ER/PgR-positive, HER2-negative (node-negative) breast cancer because it independently identifies patients with good outcomes on endocrine therapy alone at 5 and 10 years of follow-up. ${ }^{37-39}$

\section{Recommendation 1.15}

If a patient has ER/PgR-positive, HER2-negative (nodepositive) breast cancer, the clinician should not use the Breast Cancer Index to guide decisions on adjuvant systemic therapy. Type: informal consensus. Evidence quality: insufficient. Strength of recommendation: strong.

Clinical interpretation of literature review. The Breast Cancer Index was developed for patients with ER/PgR-positive, HER2negative (node-negative) breast cancer. Data are not available to support the use of the Breast Cancer Index in node-positive breast cancer.

\section{Recommendation 1.16}

If a patient has HER2-positive breast cancer or TN breast cancer, the clinician should not use the Breast Cancer Index to guide decisions on adjuvant systemic therapy. Type: informal consensus. Evidence quality: insufficient. Strength of recommendation: strong.

Clinical interpretation of literature review. The Breast Cancer Index was developed for patients with ER/PgR-positive, HER2negative (node-negative) breast cancer. Data are not available to support the use of the Breast Cancer Index in HER2-positive breast cancer or TN breast cancer.

\section{Recommendation 1.17}

If a patient has ER/PgR-positive, HER2-negative (nodepositive or node-negative) breast cancer, the clinician should not use the five-protein assay (Mammostrat; Clarient, a GE Healthcare company, Aliso Viejo, CA) to guide decisions on adjuvant systemic therapy. Type: evidence based. Evidence quality: intermediate. Strength of recommendation: moderate.

Clinical interpretation of literature review. Mammostrat has been shown to have prognostic value in ER/PgR-positive breast cancer, but the proportion of patients who were recurrence free at 10 years was only $85 \%$ in the low-risk subgroup, and a statistically significant benefit of chemotherapy was found in that subgroup. ${ }^{40}$ Furthermore, in an analysis of the prediction of adjuvant chemotherapy benefit in ER/PgR-positive breast cancer, a strong qualitative interaction was not observed, and the test for interaction was not significant. Therefore, the evidence is insufficient to recommend use of the five-protein assay to decide whether a patient should receive adjuvant chemotherapy. 


\section{Recommendation 1.18}

If a patient has HER2-positive breast cancer or TN breast cancer, the clinician should not use the five-protein assay (Mammostrat) to guide decisions on adjuvant systemic therapy. Type: informal consensus. Evidence quality: insufficient. Strength of recommendation: strong.

Clinical interpretation of literature review. Mammostrat was developed for ER/PgR-positive breast cancer. Data are not available to support the use of the five-protein assay in HER2-positive breast cancer or $\mathrm{TN}$ breast cancer.

\section{Recommendation 1.19}

If a patient has ER/PgR-positive, HER2-negative (nodepositive or node-negative) breast cancer, the clinician should not use the immunohistochemistry 4 (IHC4) assay to guide decisions on adjuvant systemic chemotherapy. Type: evidence based. Evidence quality: intermediate. Strength of recommendation: moderate.

Clinical interpretation of literature review. IHC4 has been tested and validated in a single institutional research laboratory that minimized preanalytic, analytic, and postanalytic variables. ${ }^{36,41}$ Although the published data are strong, the test has not been shown to be sufficiently reproducible in other academic or clinical laboratories to recommend it for broad clinical use. Hence, it did not meet evidence criteria for analytic validity, despite evidence for clinical utility.

\section{Recommendation 1.20}

If a patient has HER2-positive breast cancer or TN breast cancer, the clinician should not use IHC4 to guide decisions on adjuvant systemic therapy. Type: informal consensus. Evidence quality: insufficient. Strength of recommendation: strong.

Clinical interpretation of literature review. IHC4 was developed for ER/PgR-positive breast cancer. Data are not available to support the use of IHC4 in HER2-positive breast cancer or TN breast cancer.

\section{Recommendation 1.21}

If a patient has ER/PgR-positive, HER2-negative (nodenegative) breast cancer, the clinician may use urokinase plasminogen activator (uPA) and plasminogen activator inhibitor type 1 (PAI-1) to guide decisions on adjuvant systemic therapy. Type: evidence based. Evidence quality: high. Strength of recommendation: weak.

Clinical interpretation of literature review. The Chemo-N0 (Prognostic and Predictive Impact of uPA/PAI-1; ClinicalTrials. gov identifier NCT01317108) trial enrolled an unselected group of patients with breast cancer. ${ }^{42}$ By trial design, low-risk patients were untreated; therefore, the study does not provide information about the benefit of endocrine therapy alone in these patients. Because the current consensus for patients with a greater than $10 \%$ risk is to recommend adjuvant endocrine therapy, the baseline risk cannot be assessed in this context. Withholding chemotherapy may not be recommended unless these data are available. Although the study met criteria for clinical utility, the strength of the recommendation for uPA and
PAI-1 is weak because no evidence supports its use in a contemporary treatment setting.

\section{Recommendation 1.22}

If a patient has HER2-positive breast cancer or TN breast cancer, the clinician should not use UPA and PAI-1 to guide decisions on adjuvant systemic therapy. Type: informal consensus. Evidence quality: insufficient. Strength of recommendation: weak.

Clinical interpretation of literature review. Because the studies that tested the clinical utility of uPA and PAI-1 were designed before adjuvant anti-HER2 therapy was approved and no separate analysis was performed on the ER/PgR-positive cohort, data are not available to support the use of uPA and PAI-1 in HER2-positive breast cancer or TN breast cancer.

\section{Recommendation $\mathbf{1 . 2 3}$}

The clinician should not use circulating tumor cells (CTCs) to guide decisions on adjuvant systemic therapy. Type: evidence based. Evidence quality: intermediate. Strength of recommendation: strong.

Clinical interpretation of literature review. CTCs have been shown to have prognostic value in breast cancer ${ }^{43,44}$; however, no data are available that demonstrate that CTC-based tests have clinical utility.

\section{Recommendation 1.24}

If a patient has ER/PgR-positive, HER2-negative (nodepositive or node-negative) breast cancer, the clinician should not use tumor-infiltrating lymphocytes (TILs) to guide decisions on adjuvant systemic therapy. Type: informal consensus. Evidence quality: insufficient. Strength of recommendation: strong.

Clinical interpretation of literature review. Data are not available to support the use of TILs in ER/PgR-positive, HER2negative breast cancer.

\section{Recommendation 1.25}

If a patient has HER2-positive breast cancer or TN breast cancer, the clinician should not use TILs to guide decisions on adjuvant systemic therapy. Type: evidence based. Evidence quality: intermediate. Strength of recommendation: strong.

Clinical interpretation of literature review. TILs have been shown to have prognostic value in HER2-positive breast cancer and TN breast cancer. ${ }^{45,46}$ Some evidence from subset analyses points to the ability of TILs to predict benefit from anthracycline-only therapy and from trastuzumab therapy in small cohorts of patients with HER2-positive disease. However, these findings represent subset analyses. Questions also remain about whether TILs are most informative when measured intratumor or in stroma. TILs have been studied in an institutional research laboratory that minimized preanalytic, analytic, and postanalytic variables. The test has not been shown to be sufficiently reproducible in a clinical laboratory or a central reference laboratory to recommend it for broad clinical use. The panel judged that the evidence is currently not sufficiently strong to recommend TILs for use in therapy selection. 


\section{Recommendation $\mathbf{1 . 2 6}$}

Ki-67 labeling index by IHC should not be used to guide choice on adjuvant chemotherapy. Type: evidence based. Evidence quality: intermediate. Strength of recommendation: moderate.

Clinical interpretation of literature review. IHC for Ki-67 analysis lacks reproducibility across laboratories and, therefore, cannot be consistently interpreted when performed in a broad range of laboratories. ${ }^{71-75} \mathrm{IHC}$ for Ki-67 is not recommended for broad clinical use to determine whether a patient should receive chemotherapy.

\section{Recommendation 1.27}

If a patient has ER/PgR-positive, HER2-negative (nodenegative) breast cancer and has had 5 years of endocrine therapy without evidence of recurrence, the clinician should not use multiparameter gene expression or protein assays (Oncotype DX, EndoPredict, PAM50, Breast Cancer Index, or IHC4) to guide decisions on extended endocrine therapy. Type: evidence based. Evidence quality: intermediate. Strength of recommendation: moderate.

Clinical interpretation of literature review. Several clinical trials have demonstrated that extended adjuvant endocrine therapy (tamoxifen or an aromatase inhibitor) beyond 5 years further reduces the risk of subsequent recurrence and death. ${ }^{27,36,37,76}$ However, extended endocrine therapy is associated with ongoing treatment-related symptoms and risk of life-threatening toxicities. Therefore, it would be of value to identify those patients with ER/PgR-positive breast cancer who are at such low risk of late recurrence that extended endocrine therapy is not indicated. These assays have analytic validity, and recent publications have suggested their clinical validity for predicting late recurrence after 5 years of endocrine therapy. ${ }^{37,76}$ Clinical validity for late recurrence has been shown in no more than one study for each of these assays, and clinical utility has not been demonstrated. ${ }^{37,76}$ There is no consensus about how low the annual risk of recurrence should be to avoid extended adjuvant endocrine therapy, and additional well-conducted studies specifically designed to establish clinical utility in this use context are needed.

\section{CLINICAL OUESTION 2}

For women with early-stage invasive breast cancer and with known ER/PgR and HER2 status, which additional biomarkers have demonstrated clinical utility to guide choice of specific drugs or regimens for adjuvant systemic therapy?

\section{Recommendation 2.1}

The clinician should not use CYP2D6 polymorphisms to guide adjuvant endocrine therapy selection. Type: evidence based. Evidence quality: intermediate. Strength of recommendation: moderate.

Clinical interpretation of literature review. The ability of polymorphisms in CYP2D6 to predict tamoxifen benefit has been extensively studied. ${ }^{47-50}$ The results of these pharmacogenomics studies have been controversial, with more recent studies being negative. At this point, data do not support the use of this marker to select patients who may or may not benefit from tamoxifen therapy.

\section{Recommendation $\mathbf{2 . 2}$}

The clinician should not use p27 expression by IHC to guide adjuvant endocrine therapy selection. Type: informal consensus. Evidence quality: low. Strength of recommendation: strong.

Clinical interpretation of literature review. Expression of p27 was tested in an institutional research laboratory that minimized preanalytic, analytic, and postanalytic variables. ${ }^{51}$ No statistically significant prognostic effect of p27 on recurrence-free survival was found. Although a statistically significant interaction occurred between adjuvant endocrine therapy and p27 status, the hazard ratios for benefit of endocrine therapy trended in the same direction in both high- and low-p27 subgroups; therefore, that the interaction may be quantitative rather than therapeutically relevant qualitatively cannot be ruled out. Evidence from only one study is not convincing with regard to a therapeutically relevant predictive effect of p27 for tamoxifen benefit. Furthermore, the test has not been shown to be sufficiently reproducible in a clinical laboratory or a central reference laboratory. The panel believed that the evidence for utility of p27 in guiding decisions about endocrine therapy was insufficient to recommend it for clinical use.

\section{Recommendation $\mathbf{2 . 3}$}

The clinician should not use Ki-67 labeling index by IHC to guide adjuvant endocrine therapy selection. Type: evidence based. Evidence quality: intermediate. Strength of recommendation: moderate.

Clinical interpretation of literature review. Ki-67 labeling index by IHC was prognostic but not significantly predictive of letrozole benefit. $^{52,53}$ Therefore, the assay is not recommended to select patients more likely to benefit from aromatase inhibitors. Ki-67 expression was tested in an institutional research laboratory that minimized preanalytic, analytic, and postanalytic variables. However, the tests have not been shown to be sufficiently reproducible in a clinical laboratory or a central reference laboratory to recommend their clinical use.

\section{Recommendation $\mathbf{2 . 4}$}

The clinician should not use microtubule-associated protein (MAP)-Tau mRNA expression or mRNA expression by IHC to guide adjuvant chemotherapy selection. Type: evidence based. Evidence quality: intermediate. Strength of recommendation: moderate.

Clinical interpretation of literature review. MAP-Tau mRNA and protein levels were prognostic but not predictive of paclitaxel benefit. ${ }^{54,55}$ Therefore, the assays are not recommended to select patients more likely to benefit from paclitaxel. MAP-Tau levels were measured in an institutional research laboratory that minimized preanalytic, analytic, and postanalytic variables. However, the tests have not been shown to be sufficiently reproducible in a clinical laboratory or a central reference laboratory to recommend their clinical use.

\section{Recommendation $\mathbf{2 . 5}$}

The clinician should not use HER1/epidermal growth factor receptor (EGFR) expression by IHC to guide adjuvant chemotherapy selection. Type: evidence based. Evidence quality: low. Strength of recommendation: moderate. 
Clinical interpretation of literature review. HER1/EGFR expression was prognostic and predictive of paclitaxel benefit by interaction analysis for DFS but not OS in one prospectiveretrospective study. ${ }^{56}$ HER1/EGFR expression was tested in an institutional research laboratory that minimized preanalytic, analytic, and postanalytic variables. However, the test has not been shown to be sufficiently reproducible in a clinical laboratory or a central reference laboratory to recommend it for clinical use. Therefore, the assay is not currently recommended to select patients more likely to benefit from paclitaxel.

\section{Recommendation 2.6}

The clinician should not use TOP2A gene amplification or TOP2A protein expression by IHC to guide adjuvant chemotherapy selection. Type: evidence based. Evidence quality: high. Strength of recommendation: moderate.

Clinical interpretation of literature review. Protein overexpression and gene amplification of TOP2A have been extensively studied for their ability to predict anthracycline benefit. ${ }^{59-62}$ The results of these studies have been controversial and biologically difficult to explain because both gain and loss of the TOP2A gene are observed as predicting benefit from anthracyclines. Data do not support the use of these assays to select patient choice of anthracycline-based therapy. Furthermore, the tests have not been shown to be sufficiently reproducible in a clinical laboratory or a central reference laboratory to recommend their clinical use.

\section{Recommendation 2.7}

The clinician should not use HER2 and TOP2A gene coamplification; CEP17 duplication; or TIMP-1, FOXP3, or p53 protein expression to guide adjuvant chemotherapy selection. Type: evidence based. Evidence quality: intermediate. Strength of recommendation: moderate.

Clinical interpretation of literature review. HER2 and TOP2A gene coamplification; CEP17 duplication; or TIMP-1, FOXP3, or p53 protein overexpression have been evaluated to predict anthracycline benefit. ${ }^{57,58,63,64,66,67}$ Results of these studies did not demonstrate that these markers are predictors of anthracycline benefit. At this point, data do not support the use of these assays to select patients who may benefit from anthracycline-based therapy. All marker assays were tested in institutional research laboratories that minimized preanalytic, analytic, and postanalytic variables. However, the tests have not been shown to be sufficiently reproducible in a clinical laboratory or a central reference laboratory to recommend their clinical use.

\section{Recommendation 2.8}

If a patient has HER2-positive breast cancer, the clinician should not use PTEN to guide adjuvant therapy selection. Type: evidence based. Evidence quality: intermediate. Strength of recommendation: moderate.

Clinical interpretation of literature review. PTEN loss has previously been linked to anti-HER2 therapy resistance. ${ }^{68}$ However, in the analysis of a large multicenter randomized trial, PTEN loss did not correlate with trastuzumab-based therapy resistance. PTEN loss was tested in an institutional research laboratory that minimized preanalytic, analytic, and postanalytic variables, but the test is one of the most difficult to optimize in a clinical or a central reference laboratory, which may preclude its clinical use. Therefore, the assay is not recommended for use in the identification of patients less likely to benefit from anti-HER2 therapies.

\section{Recommendation 2.9}

If a patient has HER2-positive breast cancer, the clinician should not use soluble HER2 levels to guide adjuvant therapy selection. Type: evidence based. Evidence quality: low. Strength of recommendation: moderate.

Clinical interpretation of literature review. Soluble HER2 was prognostic but not predictive of trastuzumab benefit. ${ }^{69}$ Therefore, the assay is not recommended to select patients more likely to benefit from anti-HER2 therapies.

\section{PATIENT AND CLINICIAN COMMUNICATION}

Clinicians should educate patients, family members, and/or caregivers about the results of pathology tests and how they use them to develop a treatment plan tailored to the biology of the cancer. Because many patients who receive a new diagnosis are under emotional stress and/or may be unaccustomed to complex medical terminology, the use of easily understood language, at an educational level that the patient can understand, is key to clear communication. Clinicians can ask patients to repeat back to them key pieces of information, provide written or recorded notes, and use visual aids to help to ensure information is effectively communicated. Patients should be given a copy of their pathology report and ER, PgR, HER2, Oncotype DX, EndoPredict, Prosigna, Breast Cancer Index, or uPA/PAI-1 test results when indicated. Clinicians should review the results with patients, discuss the test interpretation or performance, and ask patients whether they have questions about the results.

Appendix Table A1, adapted from Wolff et al, ${ }^{3}$ offers clinicians discussion points for communicating about the use of biomarkers to guide therapy decision for patients with early-stage invasive breast cancer. In addition, information on health literacy can be found at www.cdc.gov/healthliteracy, and further details on the use of numbers to explain risk can be found at www.cdc.gov/ healthliteracy/numeracy-course/index.html.

\section{HEALTH DISPARITIES}

Although ASCO clinical practice guidelines represent expert recommendations on best practices in disease management to provide the highest level of cancer care, many patients have limited access to medical care. Racial and ethnic disparities in health care contribute significantly to this problem in the United States. Patients with cancer who are members of racial/ethnic minorities suffer disproportionately from comorbidities, experience more substantial obstacles to receiving care, are more likely to be uninsured, and are at greater risk of receiving inadequate care than other Americans. ${ }^{77-80}$ Many other patients lack access to 
care because of their geographic location and distance from appropriate treatment facilities. Awareness of these disparities in access to care should be considered in the context of this clinical practice guideline, and health care providers should strive to deliver the highest level of cancer care to these vulnerable populations.

The biomarker distribution of ER/PgR expression and HER2 status can vary across tumors in patients of various ethnic and racial backgrounds. ${ }^{81}$ In addition to the biologic variability of ER, PgR, and HER2 expression in breast cancer, disparities exist in the frequency of biomarker testing in certain populations and in what is covered by health insurance. ${ }^{82}$ The literature search performed for this guideline did not identify studies that examined the clinical utility of biomarkers across ethnic, racial, or socioeconomic backgrounds.

\section{MULTIPLE CHRONIC CONDITIONS}

Creating evidence-based recommendations to inform treatment of patients with additional chronic conditions, a situation in which the patient may have two or more such conditions (referred to as multiple chronic conditions [MCCs]), is challenging. Patients with MCCs are a complex and heterogeneous population, which makes it difficult to account for all the possible permutations to develop specific recommendations for care. In addition, the best available evidence for treating specific conditions, such as cancer, is often from clinical trials in which study selection criteria may exclude patients with MCCs to avoid potential interaction effects or confounding of results associated with MCCs. As a result, the reliability of outcome data from these studies may be limited, which thereby creates constraints for expert groups to make recommendations for care.

Because many patients for whom guideline recommendations apply present with MCCs, any treatment plan must take into account the complexity and uncertainty created by the presence of MCCs and highlight the importance of shared decision making with regard to guideline use and implementation. Therefore, in consideration of recommended care for the target condition, clinicians should review all of the patient's other chronic conditions and take those conditions into account when formulating treatment and follow-up plans.

In light of these considerations, practice guidelines should provide information on how to apply the recommendations for patients with MCCs, perhaps as a qualifying statement for recommended care. This may mean that some or all of the recommended care options are modified or not applied as determined by best practice in consideration of MCCs.

\section{GUIDELINE IMPLEMENTATION}

ASCO guidelines are developed for implementation across health care settings. Barriers to implementation include the need to increase awareness of the guideline recommendations among front-line practitioners, cancer survivors, and caregivers and to provide adequate services in the face of limited resources. The Bottom Line Box facilitates implementation of the present recommendations. This guideline will be distributed widely through the ASCO Practice Guideline Implementation Network. ASCO guidelines are posted on the ASCO Web site and most often published in Journal of Clinical Oncology and Journal of Oncology Practice.

\section{LIMITATION OF THE RESEARCH}

Conclusions on prognostic and predictive biomarkers in early-stage invasive breast cancer are limited by the lack of prospective confirmatory studies; findings of insufficient clinical utility; and, in many cases, a lack of data on clinical validity and reproducibility of assays. The expert panel awaits the completion and publication of several randomized trials to establish the clinical utility of some of these assays. Extensive research is needed to validate some of the biomarker candidates described and to identify promising new biomarkers. ASCO believes that cancer clinical trials are vital to inform medical decisions and improve cancer care and that all patients should have the opportunity to participate.

\section{FUTURE DIRECTIONS}

Research is needed in all areas in the guideline to continue to refine and redefine clinical utility of specific biomarkers. Inclusion of biomarker investigations at the beginning of clinical trials during conception and design and prospective or prospective-retrospective studies that validate the clinical utility of biomarker candidates are important to allow selection of therapy for early-stage invasive breast cancer. Research also is needed to better understand the impact of age, race/ethnicity, and health disparities on the prognostic and predictive value of biomarker $\mathrm{r}$ candidates.

\section{ADDITIONAL RESOURCES}

More information, which includes Data Supplements with additional evidence tables, a Methodology Supplement with information about evidence quality and strength of recommendations, slide sets, and clinical tools and resources, is available at www.asco. org/guidelines/adjuvantbreastmarkers and www.asco.org/guidelineswiki. Patient information is available at www.cancer.net.

\section{AUTHORS' DISCLOSURES OF POTENTIAL CONFLICTS} OF INTEREST

Disclosures provided by the authors are available with this article at www.jco.org.

\section{AUTHOR CONTRIBUTIONS}

Manuscript writing: All authors

Final approval of manuscript: All authors 


\section{REFERENCES}

1. Harris L, Fritsche H, Mennel R, et al: American Society of Clinical Oncology: American Society of Clinical Oncology 2007 update of recommendations for the use of tumor markers in breast cancer. J Clin Oncol 25:5287-5312, 2007

2. Wolff AC, Hammond ME, Schwartz JN, et al: American Society of Clinical Oncology/College of American Pathologists: American Society of Clinical Oncology/College of American Pathologists guideline recommendations for human epidermal growth factor receptor 2 testing in breast cancer. Arch Pathol Lab Med 131:18-43, 2007

3. Wolff $A C$, Hammond ME, Hicks DG, et al: American Society of Clinical Oncology; College of American Pathologists: Recommendations for human epidermal growth factor receptor 2 testing in breast cancer: American Society of Clinical Oncology/ College of American Pathologists clinical practice guideline update. J Clin Oncol 31:3997-4013, 2013

4. Hammond ME, Hayes DF, Dowsett M, et al: American Society of Clinical Oncology/College of American Pathologists guideline recommendations for immunohistochemical testing of estrogen and progesterone receptors in breast cancer. Arch Pathol Lab Med 134:907-922, 2010

4a. Van Poznak C, Somerfield MR, Bast RC, et al: The use of biomarkers to guide decisions on systemic therapy for women with metastatic breast cancer: American Society of Clinical Oncology clinical practice guideline. J Clin Oncol, doi: 10.1200/ JCO.2015.61.1459

5. Hayes DF, Bast RC, Desch CE, et al: Tumor marker utility grading system: A framework to evaluate clinical utility of tumor markers. J Natl Cancer Inst 88:1456-1466, 1996

6. Ginsburg GS, Kuderer NM: Comparative effectiveness research, genomics-enabled personalized medicine, and rapid learning health care: A common bond. J Clin Oncol 30:4233-4242, 2012

7. Hayes DF, Allen J, Compton C, et al: Breaking a vicious cycle. Sci Transl Med 5:196cm6, 2013

8. McShane LM, Hayes DF: Publication of tumor marker research results: The necessity for complete and transparent reporting. J Clin Oncol 30:4223-4232, 2012

9. Simon RM, Paik S, Hayes DF: Use of archived specimens in evaluation of prognostic and predictive biomarkers. J Natl Cancer Inst 101: 1446-1452, 2009

10. Janes $H$, Pepe MS, Bossuyt PM, et al: Measuring the performance of markers for guiding treatment decisions. Ann Intern Med 154:253-259, 2011

11. Kaufmann M, Pusztai L; Biedenkopf Expert Panel Members: Use of standard markers and incorporation of molecular markers into breast cancer therapy: Consensus recommendations from an International Expert Panel. Cancer 117:1575-1582, 2011

12. Polley MY, Freidlin B, Korn EL, et al: Statistical and practical considerations for clinical evaluation of predictive biomarkers. J Natl Cancer Inst 105: 1677-1683, 2013

13. Teutsch SM, Bradley LA, Palomaki GE, et al: EGAPP Working Group: The evaluation of genomic applications in practice and prevention (EGAPP) initiative: Methods of the EGAPP Working Group. Genet Med 11:3-14, 2009

14. Botkin JR, Teutsch SM, Kaye Cl, et al: EGAPP Working Group: Outcomes of interest in evidencebased evaluations of genetic tests. Genet Med 12: 228-235, 2010
15. Micheel CM, Nass SJ, Omenn GS (eds): Evolution of Translational Omics: Lessons Learned and the Path Forward. Washington, DC, National Academies Press, 2012

16. Wolff AC, Hammond ME, Hicks DG, et al: American Society of Clinical Oncology; College of American Pathologists: Recommendations for human epidermal growth factor receptor 2 testing in breast cancer: American Society of Clinical Oncology/ College of American Pathologists clinical practice guideline update. Arch Pathol Lab Med 138:241-256, 2014

17. Peto R, Davies C, Godwin J, et al: Early Breast Cancer Trialists' Collaborative Group (EBCTCG): Comparisons between different polychemotherapy regimens for early breast cancer: Meta-analyses of long-term outcome among 100,000 women in 123 randomised trials. Lancet 379:432-444, 2012

18. Sargent DJ, Conley BA, Allegra C, et al: Clinical trial designs for predictive marker validation in cancer treatment trials. J Clin Oncol 23 2020-2027, 2005

19. Freidlin B, McShane LM, Korn EL: Randomized clinical trials with biomarkers: Design issues. J Natl Cancer Inst 102:152-160, 2010

20. Paik S, Shak S, Tang G, et al: A multigene assay to predict recurrence of tamoxifen-treated, node-negative breast cancer. N Engl J Med 351: 2817-2826, 2004

21. Paik S, Tang G, Shak S, et al: Gene expression and benefit of chemotherapy in women with nodenegative, estrogen receptor-positive breast cancer. J Clin Oncol 24:3726-3734, 2006

22. Dowsett M, Cuzick J, Wale C, et al: Prediction of risk of distant recurrence using the 21-gene recurrence score in node-negative and node-positive postmenopausal patients with breast cancer treated with anastrozole or tamoxifen: A TransATAC study. J Clin Oncol 28:1829-1834, 2010

23. Albain KS, Barlow WE, Shak $S$, et al: Breast Cancer Intergroup of North America: Prognostic and predictive value of the 21-gene recurrence score assay in postmenopausal women with node-positive, oestrogen-receptor-positive breast cancer on chemotherapy: A retrospective analysis of a randomised trial. Lancet Oncol 11:55-65, 2010

24. Filipits $M$, Rudas $M$, Jakesz $R$, et al: EP Investigators: A new molecular predictor of distant recurrence in ER-positive, HER2-negative breas cancer adds independent information to conventional clinical risk factors. Clin Cancer Res 17:6012-6020, 2011

25. Dubsky $P$, Filipits $M$, Jakesz $R$, et al: Austrian Breast and Colorectal Cancer Study Group (ABCSG): EndoPredict improves the prognostic classification derived from common clinical guidelines in ERpositive, HER2-negative early breast cancer. Ann Oncol 24:640-647, 2013

26. Martin M, Brase JC, Calvo L, et al: Clinical validation of the EndoPredict test in node-positive, chemotherapy-treated ER+/HER2- breast cancer patients: Results from the GEICAM 9906 trial. Breast Cancer Res 16:R38, 2014

27. Dubsky P, Brase JC, Jakesz R, et al: Austrian Breast and Colorectal Cancer Study Group (ABCSG): The EndoPredict score provides prognostic information on late distant metastases in ER+/HER2 breast cancer patients. Br J Cancer 109:2959-2964, 2013

28. Buyse $M$, Loi $S$, van't Veer $L$, et al: TRANSBIG Consortium: Validation and clinical utility of a 70-gene prognostic signature for women with node- negative breast cancer. J Natl Cancer Inst 98: 1183-1192, 2006

29. Mook S, Schmidt MK, Viale G, et al: TRANSBIG Consortium: The 70-gene prognosis-signature predicts disease outcome in breast cancer patients with 1-3 positive lymph nodes in an independent validation study. Breast Cancer Res Treat 116: 295-302, 2009

30. Bueno-de-Mesquita JM, Linn SC, Keijzer R, et al: Validation of 70-gene prognosis signature in node-negative breast cancer. Breast Cancer Res Treat 117:483-495, 2009

31. Knauer $M$, Cardoso $F$, Wesseling $J$, et al: Identification of a low-risk subgroup of HER-2positive breast cancer by the 70-gene prognosis signature. Br J Cancer 103:1788-1793, 2010

32. Knauer M, Mook S, Rutgers EJ, et al: The predictive value of the 70-gene signature for adjuvant chemotherapy in early breast cancer. Breast Cancer Res Treat 120:655-661, 2010

33. Drukker CA, van Tinteren H, Schmidt MK, et al: Long-term impact of the 70-gene signature on breast cancer outcome. Breast Cancer Res Treat 143: 587-592, 2014

34. Filipits M, Nielsen TO, Rudas M, et al: Austrian Breast and Colorectal Cancer Study Group: The PAM50 risk-of-recurrence score predicts risk for late distant recurrence after endocrine therapy in postmenopausal women with endocrine-responsive early breast cancer. Clin Cancer Res 20:1298-1305, 2014

35. Gnant $M$, Filipits $M$, Greil $R$, et al: Austrian Breast and Colorectal Cancer Study Group: Predicting distant recurrence in receptor-positive breast cancer patients with limited clinicopathological risk: Using the PAM50 risk of recurrence score in 1478 postmenopausal patients of the ABCSG-8 trial treated with adjuvant endocrine therapy alone. Ann Oncol 25: 339-345, 2014

36. Dowsett M, Sestak I, Lopez-Knowles E, et al: Comparison of PAM50 risk of recurrence score with Oncotype DX and IHC4 for predicting risk of distant recurrence after endocrine therapy. J Clin Oncol 31: 2783-2790, 2013

37. Sgroi DC, Sestak I, Cuzick J, et al: Prediction of late distant recurrence in patients with oestrogenreceptor-positive breast cancer: A prospective comparison of the breast-cancer index (BCl) assay, 21-gene recurrence score, and IHC4 in the TransATAC study population. Lancet Oncol 14:1067-1076, 2013

38. Jerevall PL, MaXJ, Li H, et al: Prognostic utility of HOXB13:IL17BR and molecular grade index in early-stage breast cancer patients from the Stockholm trial. Br J Cancer 104:1762-1769, 2011

39. Zhang $Y$, Schnabel CA, Schroeder BE, et al: Breast cancer index identifies early-stage estrogen receptor-positive breast cancer patients at risk for early-and late-distant recurrence. Clin Cancer Res 19: 4196-4205, 2013

40. Ross DT, Kim CY, Tang G, et al: Chemosensitivity and stratification by a five monoclonal antibody immunohistochemistry test in the NSABP B14 and B20 trials. Clin Cancer Res 14:6602-6609, 2008

41. Cuzick J, Dowsett M, Pineda S, et al: Prognostic value of a combined estrogen receptor, progesterone receptor, Ki-67, and human epidermal growth factor receptor 2 immunohistochemical score and comparison with the Genomic Health recurrence score in early breast cancer. J Clin Oncol 29: 4273-4278, 2011

42. Harbeck N, Schmitt M, Meisner C, et al: Chemo-N 0 Study Group: Ten-year analysis of the 
prospective multicentre Chemo-NO trial validates American Society of Clinical Oncology (ASCO)recommended biomarkers UPA and PAl-1 for therapy decision making in node-negative breast cancer patients. Eur J Cancer 49:1825-1835, 2013

43. Rack B, Schindlbeck C, Jückstock J, et al: SUCCESS Study Group: Circulating tumor cells predict survival in early average-to-high risk breast cancer patients. J Natl Cancer Inst 106:dju066, 2014

44. Lucci A, Hall CS, Lodhi AK, et al: Circulating tumour cells in non-metastatic breast cancer: A prospective study. Lancet Oncol 13:688-695, 2012

45. Loi S, Michiels S, Salgado R, et al: Tumor infiltrating lymphocytes are prognostic in triple negative breast cancer and predictive for trastuzumab benefit in early breast cancer: Results from the FinHER trial. Ann Oncol 25:1544-1550, 2014

46. Loi S, Sirtaine N, Piette F, et al: Prognostic and predictive value of tumor-infiltrating lymphocytes in a phase III randomized adjuvant breast cancer trial in node-positive breast cancer comparing the addition of docetaxel to doxorubicin with doxorubicin-based chemotherapy: BIG 02-98. J Clin Oncol 31:860-867, 2013

47. Province MA, Goetz MP, Brauch $H$, et al: International Tamoxifen Pharmacogenomics Consortium: CYP2D6 genotype and adjuvant tamoxifen: Meta-analysis of heterogeneous study populations. Clin Pharmacol Ther 95:216-227, 2014

48. Goetz MP, Suman VJ, Hoskin $T L$, et al: CYP2D6 metabolism and patient outcome in the Austrian Breast and Colorectal Cancer Study Group trial (ABCSG) 8. Clin Cancer Res 19:500-507, 2013

49. Regan MM, Leyland-Jones $B$, Bouzyk M, et al: Breast International Group (BIG) 1-98 Collaborative Group: CYP2D6 genotype and tamoxifen response in postmenopausal women with endocrine-responsive breast cancer: The Breast International Group 1-98 trial. J Natl Cancer Inst 104:441-451, 2012

50. Rae JM, Drury $S$, Hayes DF, et al: ATAC Trialists: CYP2D6 and UGT2B7 genotype and risk of recurrence in tamoxifen-treated breast cancer patients. J Natl Cancer Inst 104:452-460, 2012

51. Stendahl M, Nilsson $S$, Wigerup $C$, et al: p27Kip1 is a predictive factor for tamoxifen treatment response but not a prognostic marker in premenopausal breast cancer patients. Int J Cancer 127: 2851-2858, 2010

52. Viale G, Giobbie-Hurder $A$, Regan MM, et al: Breast International Group Trial 1-98: Prognostic and predictive value of centrally reviewed Ki-67 labeling index in postmenopausal women with endocrineresponsive breast cancer: Results from Breast International Group Trial 1-98 comparing adjuvant tamoxifen with letrozole. J Clin Oncol 26:5569-5575, 2008

53. Viale G, Regan MM, Dell'Orto P, et al: BIG 1-98 Collaborative and International Breast Cancer Study Groups: Which patients benefit most from adjuvant aromatase inhibitors? Results using a composite measure of prognostic risk in the BIG $1-98$ randomized trial. Ann Oncol 22:2201-2207, 2011

54. Pentheroudakis G, Kalogeras KT, Wirtz RM, et al: Gene expression of estrogen receptor, progesterone receptor and microtubule-associated protein Tau in high-risk early breast cancer: A quest for molecular predictors of treatment benefit in the context of a Hellenic Cooperative Oncology Group trial. Breast Cancer Res Treat 116:131-143, 2009

55. Pusztai $L$, Jeong JH, Gong $Y$, et al: Evaluation of microtubule-associated protein-Tau expression as a prognostic and predictive marker in the NSABP-B 28 randomized clinical trial. J Clin Oncol 27: 4287-4292, 2009

56. Tzaida O, Gogas H, Dafni U, et al: Evaluation of the prognostic and predictive value of HER-1/EGFR in breast cancer patients participating in a randomized study with dose-dense sequential adjuvant chemotherapy. Oncology 72:388-396, 2007

57. Di Leo A, Desmedt C, Bartlett JMS, et al: HER2/TOP2A Meta-analysis Study Group: HER2 and TOP2A as predictive markers for anthracyclinecontaining chemotherapy regimens as adjuvant treatment of breast cancer: A meta-analysis of individual patient data. Lancet Oncol 12:1134-1142, 2011

58. Tanner M, Isola J, Wiklund T, et al: Scandinavian Breast Group Trial 9401: Topoisomerase Ilalpha gene amplification predicts favorable treatment response to tailored and dose-escalated anthracycline-based adjuvant chemotherapy in HER-2/neu-amplified breast cancer: Scandinavian Breast Group Trial 9401. J Clin Oncol 24:2428-2436, 2006

59. Du Y, Zhou Q, Yin W, et al: The role of topoisomerase $\| \alpha$ in predicting sensitivity to anthracyclines in breast cancer patients: A meta-analysis of published literatures. Breast Cancer Res Treat 129: 839-848, 2011

60. Hannemann $\mathrm{J}$, Kristel $\mathrm{P}$, van Tinteren $\mathrm{H}$, et al: Molecular subtypes of breast cancer and amplification of topoisomerase II alpha: Predictive role in dose intensive adjuvant chemotherapy. $\mathrm{Br} \mathrm{J}$ Cancer 95: 1334-1341, 2006

61. Nielsen KV, Ejlertsen $B$, Møller $S$, et al: The value of TOP2A gene copy number variation as a biomarker in breast cancer: Update of DBCG trial 89D. Acta Oncol 47:725-734, 2008

62. O'Malley FP, Chia S, Tu D, et al: Topoisomerase Il alpha protein and responsiveness of breast cancer to adjuvant chemotherapy with CEF compared to CMF in the NCIC CTG randomized MA.5 adjuvant trial. Breast Cancer Res Treat 128:401-409, 2011

63. Pritchard KI, Munro A, O'Malley FP, et al: Chromosome 17 centromere (CEP17) duplication as a predictor of anthracycline response: Evidence from the NCIC Clinical Trials Group (NCIC CTG) MA.5 trial. Breast Cancer Res Treat 131:541-551, 2012

64. Ladoire S, Mignot G, Dalban C, et al: FOXP3 expression in cancer cells and anthracyclines efficacy in patients with primary breast cancer treated with adjuvant chemotherapy in the phase III UNICANCERPACS 01 trial. Ann Oncol 23:2552-2561, 2012

65. Conforti R, Boulet T, Tomasic G, et al: Breast cancer molecular subclassification and estrogen receptor expression to predict efficacy of adjuvant anthracyclines-based chemotherapy: A biomarker study from two randomized trials. Ann Oncol 18: 1477-1483, 2007

66. Hertel PB, Tu D, Ejlertsen B, et al: TIMP-1 in combination with HER2 and TOP2A for prediction of benefit from adjuvant anthracyclines in high-risk breast cancer patients. Breast Cancer Res Treat 132:225-234, 2012

67. Lara JF, Thor AD, Dressler LG, et al: Cancer and Leukemia Group B: p53 expression in nodepositive breast cancer patients: Results from the Cancer and Leukemia Group B 9344 Trial (159905). Clin Cancer Res 17:5170-5178, 2011

68. Perez EA, Dueck AC, McCullough AE, et al: Impact of PTEN protein expression on benefit from adjuvant trastuzumab in early-stage human epidermal growth factor receptor 2-positive breast cancer in the North Central Cancer Treatment Group N9831 trial. J Clin Oncol 31:2115-2122, 2013

69. Moreno-Aspitia A, Hillman DW, Dyar SH, et al: Soluble human epidermal growth factor receptor 2 (HER2) levels in patients with HER2-positive breast cancer receiving chemotherapy with or without trastuzumab: Results from North Central Cancer Treatment Group Adjuvant Trial N9831. Cancer 119: 2675-2682, 2013

70. Hayes DF: Targeting adjuvant chemotherapy: A good idea that needs to be proven! J Clin Oncol 30: 1264-1267, 2012

71. Early Breast Cancer Trialists' Collaborative Group (EBCTCG): Effects of chemotherapy and hormonal therapy for early breast cancer on recurrence and 15-year survival: An overview of the randomised trials. Lancet 365:1687-1717, 2005

72. Goldhirsch A, Winer EP, Coates AS, et al: Personalizing the treatment of women with early breast cancer: Highlights of the St Gallen International Expert Consensus on the Primary Therapy of Early Breast Cancer 2013. Ann Oncol 24:2206-2223, 2013

73. Yerushalmi R, Woods R, Ravdin PM, et al: Ki67 in breast cancer: Prognostic and predictive potential. Lancet Oncol 11:174-183, 2010

74. Dowsett M, Nielsen TO, A'Hern R, et al: International Ki-67 in Breast Cancer Working Group: Assessment of Ki67 in breast cancer: Recommendations from the International Ki67 in Breast Cancer Working Group. J Natl Cancer Inst 103: 1656-1664, 2011

75. Polley MYC, Leung SCY, McShane LM, et al: International Ki67 in Breast Cancer Working Group of the Breast International Group and North American Breast Cancer Group: An international Ki67 reproducibility study. J Natl Cancer Inst 105:1897-1906, 2013

76. Sestak I, Cuzick J, Dowsett M, et al: Prediction of late distant recurrence after 5 years of endocrine treatment: A combined analysis of patients from the Austrian Breast and Colorectal Cancer Study Group 8 and Arimidex, Tamoxifen Alone or in Combination randomized trials using the PAM50 risk of recurrence score. J Clin Oncol 33:916-922, 2015

77. Howlader N, Noone AM, Krapcho M, et al: Cancer Facts \& Figures for African Americans 20132014. Atlanta, GA American Cancer Society, 2013

78. American Geriatrics Society Expert Panel on the Care of Older Adults with Multimorbidity: Patientcentered care for older adults with multiple chronic conditions: A stepwise approach from the American Geriatrics Society Expert Panel on the Care of Older Adults with Multimorbidity. J Am Geriatr Soc 60: 1957-1968, 2012

79. US Cancer Statistics Working Group: United States Cancer Statistics: 1999-2011 Incidence and mortality web-based report. Atlanta, GA, US Department of Health and Human Services, Centers for Disease Control and Prevention and National Cancer Institute. http://www.cdc.gov/uscs

80. Mead $H$, Cartwright-Smith $L$, Jones $K$, et al: Racial and ethnic disparities in U.S. health care: A chartbook, 2008. http://www.commonwealthfund. org/publications/chartbooks/2008/mar/racial-and-ethnicdisparities-in-u-s-health-care-a-chartbook

81. Singh $M$, Ding $Y$, Zhang $L Y$, et al: Distinct breast cancer subtypes in women with early-onset disease across races. Am J Cancer Res 4:337-352, 2014

82. Stark A, Kucera G, Lu M, et al: Influence of health insurance status on inclusion of HER-2/neu testing in the diagnostic workup of breast cancer patients. Int J Qual Health Care 16:517-521, 2004 


\section{Affiliations}

Lyndsay N. Harris, Case Western Reserve University, Cleveland, OH; Nofisat Ismaila, American Society of Clinical Oncology, Alexandria, VA; Lisa M. McShane, National Cancer Institute, Bethesda, MD; Fabrice Andre, Institute Gustave Roussy, Paris, France; Deborah E. Collyar, Patient Advocates in Research; Elizabeth H. Hammond, University of Utah and Intermountain Health Care, Salt Lake City, UT; Ana M. Gonzalez-Angulo and Robert C. Bast, The University of Texas MD Anderson Cancer Center, Houston; Robert G.Mennel, Baylor University Medical Center and Texas Oncology PA, Dallas, TX; Nicole M. Kuderer, University of Washington Medical Center, Seattle, WA; Minetta C. Liu, Mayo Clinic College of Medicine, Rochester, MN; and Catherine Van Poznak and Daniel F. Hayes, University of Michigan Comprehensive Cancer Center, Ann Arbor, MI. 


\section{AUTHORS' DISCLOSURES OF POTENTIAL CONFLICTS OF INTEREST}

Use of Biomarkers to Guide Decisions on Adjuvant Systemic Therapy for Women With Early-Stage Invasive Breast Cancer: American Society of Clinical Oncology Clinical Practice Guideline

The following represents disclosure information provided by authors of this manuscript. All relationships are considered compensated. Relationships are self-held unless noted. I = Immediate Family Member, Inst = My Institution. Relationships may not relate to the subject matter of this manuscript. For more information about ASCO's conflict of interest policy, please refer to www.asco.org/rwc or jco.ascopubs.org/site/ifc.

Lyndsay N. Harris

Research Funding: Philips Research

Nofisat Ismaila

Employment: GlaxoSmithKline (I)

Stock or Other Ownership: GlaxoSmithKline (I)

Travel, Accommodations, Expenses: GlaxoSmithKline (I)

\section{Lisa M. McShane}

No relationship to disclose

Fabrice Andre

Research Funding: AstraZeneca (Inst), Novartis (Inst), Pfizer (Inst), Eisai (Inst), Eli Lilly (Inst), Servier (Inst)

Travel, Accommodations, Expenses: Novartis, Roche, GlaxoSmithKline

Deborah E. Collyar

Travel, Accommodations, Expenses: Merck

Ana M. Gonzalez-Angulo

No relationship to disclose

Elizabeth H. Hammond

No relationship to disclose

\section{Nicole M. Kuderer}

Consulting or Advisory Role: Daiichi Sankyo, Janssen Pharmaceuticals (a Johnson \& Johnson co.), Hospira

Research Funding: Amgen (I)

Travel, Accommodations, Expenses: Daiichi Sankyo, Janssen

Pharmaceuticals (a Johnson \& Johnson co.), Hospira

\section{Minetta C. Liu}

Research Funding: Eisai (Inst), Seattle Genetics (Inst), Celgene (Inst), Veridex (Inst), Clearbridge Biomedics (Inst), Novartis (Inst), Genetech (Inst)

\author{
Robert G. Mennel \\ Employment: Texas Oncology
}

Catherine Van Poznak

Research Funding: Bayer AG (Inst)

Patents, Royalties, Other Intellectual Property: UpToDate

Robert C. Bast

Research Funding: Arrien Pharmaceuticals

Patents, Royalties, Other Intellectual Property: Fujirebio Diagnostics

Travel, Accommodations, Expenses: Roche

Daniel F. Hayes

Stock or Other Ownership: Oncimmune, Inbiomotion

Honoraria: Eli Lilly

Consulting or Advisory Role: Pfizer, Janssen Diagnostics (unpaid)

Research Funding: Janssen Research \& Development (Inst), AstraZeneca (Inst), Puma Biotechnology (Inst), Pfizer (Inst), Eli Lilly (Inst)

Patents, Royalties, Other Intellectual Property: Royalties from licensed technology; Diagnosis and Treatment of Breast Cancer (Patent No. US 8,790,878 B2; Date of patent: July 29, 2014; Applicant proprietor: University of Michigan; Daniel F. Hayes is designated as inventor/ coinventor); Circulating Tumor Cell Capturing Techniques and Devices (Patent No. US 8,951,484 B2; Date of patent: February 10, 2015; Applicant proprietor: University of Michigan; Daniel F. Hayes is designated as inventor/coinventor); A Method for Predicting Progression Free and Overall Survival at Each Follow Up Timepoint During Therapy of Metastatic Breast Cancer Patients Using Circulating Tumor Cells (Patent No. 05725638.0-1223-US2005008602)

Travel, Accommodations, Expenses: Janssen Diagnostics 


\section{Acknowledgment}

We thank the Clinical Practice Guideline Committee for its thoughtful review of and insightful comments on this guideline document.

\section{Appendix}

\begin{tabular}{|c|c|}
\hline Key Point & Action \\
\hline $\begin{array}{l}\text { Explain the importance of determining the biologic } \\
\text { characteristics of breast cancer. }\end{array}$ & $\begin{array}{l}\text { Patients should understand that the most common biologic } \\
\text { tests are those for ER, PgR, and HER2 and that testing for } \\
\text { these markers is important to select an appropriate } \\
\text { treatment. } \\
\text { Patients should understand that assay validity and clinical } \\
\text { validity of a biomarker are important steps in test } \\
\text { development but that, by themselves, they are insufficient to } \\
\text { guide treatment decisions outside of a clinical trial. } \\
\text { The patient should be aware of the balance between benefit } \\
\text { and harm and the importance of a biomarker showing clinical } \\
\text { utility before being used in routine care. } \\
\text { Patients and clinicians should understand that a wide range of } \\
\text { biomarker tests are technically possible. In addition to ER, } \\
\text { PgR, and HER2, only Oncotype DX, EndoPredict, Prosigna, } \\
\text { Breast Cancer Index, and uPA/PAI-1 have demonstrated } \\
\text { clinical utility for early-stage invasive breast cancer. }\end{array}$ \\
\hline $\begin{array}{l}\text { Explain the importance of ER, PgR, HER2, Oncotype DX, } \\
\text { EndoPredict, Prosigna, Breast Cancer Index, or uPA/PAl-1 } \\
\text { testing. }\end{array}$ & $\begin{array}{l}\text { Patients should understand that in addition to ER, PgR, and } \\
\text { HER2, Oncotype DX, EndoPredict, Prosigna, Breast Cancer } \\
\text { Index, or uPA/PAl-1 status determines whether their } \\
\text { prognosis is sufficiently good that chemotherapy might not } \\
\text { be recommended. }\end{array}$ \\
\hline $\begin{array}{l}\text { Explain the types of tests used to determine ER, PgR, HER2, } \\
\text { Oncotype DX, EndoPredict, Prosigna, Breast Cancer Index, or } \\
\text { uPA/PAl-1 status. }\end{array}$ & $\begin{array}{l}\text { Patients should understand that different testing methods are } \\
\text { used to detect ER, PgR, HER2, Oncotype DX, EndoPredict, } \\
\text { Prosigna, Breast Cancer Index, or uPA/PAl-1 status, which } \\
\text { include evaluation of protein overexpression or the presence } \\
\text { of genetic alterations. }\end{array}$ \\
\hline $\begin{array}{l}\text { Explain the interpretation of the ER, PgR, HER2, Oncotype DX, } \\
\text { EndoPredict, Prosigna, Breast Cancer Index, or uPA/PAI-1 } \\
\text { test results. }\end{array}$ & $\begin{array}{l}\text { Patients should understand that although most test results are } \\
\text { definitively positive or negative, there are equivocal results } \\
\text { that require additional testing with an alternative test or the } \\
\text { same or alternative test on a different portion of the same } \\
\text { specimen (different block). Sometimes, the oncologist or } \\
\text { pathologist may recommend additional testing with a } \\
\text { different type of tumor specimen (eg, surgical excision } v \text { core } \\
\text { biopsy), if available. } \\
\text { Unfortunately, some results remain indeterminate or } \\
\text { inconsistent with other histopathologic findings. In such } \\
\text { cases, a final treatment decision to consider treatment with } \\
\text { ER-, PgR-, or HER2-targeted therapy or therapy guided by } \\
\text { Oncotype DX, EndoPredict, Prosigna, Breast Cancer Index, or } \\
\text { uPAPAl-1 should be made after consultation between the } \\
\text { pathologist and oncologist and a discussion with the patient. }\end{array}$ \\
\hline $\begin{array}{l}\text { Explain that ER, PgR, HER2, and Oncotype DX, EndoPredict, } \\
\text { Prosigna, Breast Cancer Index, or uPA/PAl-1 testing } \\
\text { guidelines exist. }\end{array}$ & $\begin{array}{l}\text { Guidelines are in place for testing biomarkers on tumor } \\
\text { specimens. Although several tests are now recommended in } \\
\text { the guidelines, only one needs to be used to guide therapy for } \\
\text { an individual patient. Although the tests are similar in nature, } \\
\text { they are not identical, and two tests on the same tissue may } \\
\text { give different results. If two or more tests are applied to the } \\
\text { same tissue, data do not exist on how to reconcile which test } \\
\text { is superior or which should be used to guide treatment } \\
\text { decisions. Patients may be referred to the ASCO Web sites } \\
\text { for additional patient-focused information. }\end{array}$ \\
\hline \multicolumn{2}{|c|}{$\begin{array}{l}\text { NOTE. Adapted with permission. }{ }^{3} \text { During discussion about the use of biomarkers to guide therapy decisions, establish how the patient prefers to receive information, } \\
\text { the patient's perceptions of the diagnosis, and tumor factors that might influence decision making; educate the patient (and family members or caregivers) about the } \\
\text { results of tests and how they are used to develop a treatment plan tailored to the biology of the cancer; and use easily understood language (at an educational level that } \\
\text { the patient can understand) to communicate clearly. } \\
\text { Abbreviations: ER, estrogen receptor; HER2, human epidermal growth factor receptor 2; PAI-1, plasminogen activator inhibitor type 1; PgR, progesterone receptor; uPA, } \\
\text { urokinase plasminogen activator. }\end{array}$} \\
\hline
\end{tabular}

